\title{
Protective effect of taraxasterol against rheumatoid arthritis by the modulation of inflammatory responses in mice
}

\author{
SHU-HUA JIANG, LI-FENG PING, FENG-YAN SUN, XIAO-LEI WANG and ZHI-JUAN SUN \\ Department of Rheumatism, Hebei Cangzhou Hospital of Integrated Traditional Chinese \\ and Western Medicine, Cangzhou, Hebei 061000, P.R. China
}

Received April 30, 2015; Accepted August 16, 2016

DOI: $10.3892 /$ etm.2016.3860

\begin{abstract}
Taraxasterol is an effective component of dandelion that has anti-inflammatory effects in vivo and in vitro. The present study was performed to explore whether taraxasterol exhibits a protective effect against rheumatoid arthritis through the modulation of inflammatory responses in mice. Eight-week-old CCR9-deficient mice were injected with a collagen II monoclonal antibody cocktail to create a rheumatoid arthritis model. In the experimental group, arthritic model mice were treated with $10 \mathrm{mg} / \mathrm{kg}$ taraxasterol once per day for 5 days. Treatment with taraxasterol significantly increased the pain thresholds and reduced the clinical arthritic scores of the mice in the experimental group compared with those of the model group. Furthermore, treatment with taraxasterol significantly suppressed tumor necrosis factor- $\alpha$, interleukin (IL) $-1 \beta$, IL-6 and nuclear factor- $\kappa \mathrm{B}$ protein expression levels compared with those in the rheumatoid arthritis model mice. Taraxasterol treatment also significantly reduced nitric oxide, prostaglandin E2 and cyclooxygenase-2 levels compared with those in the rheumatoid arthritis model group. These observations indicate that the protective effect of taraxasterol against rheumatoid arthritis is mediated via the modulation of inflammatory responses in mice.
\end{abstract}

\section{Introduction}

Rheumatoid arthritis is a type of systemic autoimmune disease, which involves symmetrical chronic inflammation of the synovial membranes, and has the following pathological characteristics: Chronic inflammation of synovial membranes, the gradual formation of pannus, cartilage and joint destruction, ankylosis and joint deformity in the advanced stage and

Correspondence to: Mr. Li-Feng Ping, Department of Rheumatism, Hebei Cangzhou Hospital of Integrated Traditional Chinese and Western Medicine, 31 Yellow River West Road, Cangzhou, Hebei 061000, P.R. China

E-mail: fysun261@163.com

Key words: taraxasterol, rheumatoid arthritis, inflammatory, nitric oxide, prostaglandin E2, cyclooxygenase-2 possible muscle atrophy (1). As a result, rheumatoid arthritis, the morbidity rate of which is $0.2-0.4 \%$, is a major cause of productivity loss and disability, and can cause great suffering and a heavy financial burden (2). Furthermore, rheumatoid arthritis is a systematic disease whose pathogenesis is unclear. However, rheumatoid arthritis is commonly considered to be induced by exogenous factors in addition to genetic susceptibility (3). Therefore, as no specific medicine for this disease has yet been developed, immunosuppressive and biological agents are widely used because of their curative abilities, despite their side-effects (4).

Dandelion is the dry grass of the Asteraceae (Compositae) family, which is used in the treatment of malignant boils, skin ulcers, acute mastitis, acute conjunctivitis, pulmonary abscess, damp-heat jaundice, heat stranguria and other inflammatory conditions, with various effects, including detoxification, detumescence, fluid removal and the induction of diuresis (5). One of the effective components of dandelion is taraxasterol, which has a molecular structure resembling that of a steroid hormone, and has been shown to have anti-inflammatory effects in vivo and in vitro (6). Therefore, the present study examined whether taraxasterol exhibits a protective effect against rheumatoid arthritis through the modulation of inflammatory responses in mice.

\section{Materials and methods}

Animals and rheumatoid arthritis induction. Eight-week-old chemokine (C-C motif) receptor 9 (CCR9)-deficient male mice ( $n=32$; weight, 20-23 g) were obtained from the Animal Resource Center of the Hebei Cangzhou Hospital of Integrated Traditional Chinese and Western Medicine (Cangzhou, China). The mice were maintained in individual cages at $21-24^{\circ} \mathrm{C}$ and $55-65 \%$ humidity with a 12 -h light-dark cycle (8:00-20:00). Food and water were provided ad libitum. The present study was conducted according to the National Institutes of Health Guide for the Care and Use of Laboratory Animals (2012). Mice were injected with a collagen II monoclonal antibody cocktail (Sigma-Aldrich, St. Louis, MO, USA) intraperitoneally (i.p.) at $5 \mathrm{mg} / \mathrm{kg}$ per mouse, as indicated (7) for 10 days. The mice were also injected with lipopolysaccharide (LPS; i.p., $100 \mu \mathrm{g}$ at day 1 or day 4; Sigma-Aldrich). Following treatment with taraxasterol, blood samples were collected from retro-orbital bleeds using 
heparin-coated glass capillaries and stored at $-80^{\circ} \mathrm{C}$ prior to analysis.

Animal grouping. The mice used in the experiment were randomly distributed into four groups: Control $(n=6)$, taraxasterol only $(n=6)$, rheumatoid arthritis model $(n=10)$ and rheumatoid arthritis + taraxasterol-treated $(n=10)$ groups. Mice of the control or rheumatoid arthritis model groups were treated with $0.5 \%$ sodium tvlose (Nanjing Chemical Reagent Co., Ltd., Nanjing, China; i.p.) once per day for 5 consecutive days following the establishment of the collagen-induced rheumatoid arthritis model. The mice of the taraxasterol only and rheumatoid arthritis + taraxasterol-treated groups were treated with $10 \mathrm{mg} / \mathrm{kg}$ taraxasterol (i.p.) once per day for 5 consecutive days. The chemical structure of taraxasterol ( $\geq 98.5 \%$ purity; Sigma-Aldrich) is shown in Fig. 1 .

Measurement of pain thresholds in the rheumatoid arthritis mouse model. The mean value of the pressure pain threshold was determined before and after modeling, and after the 5-day treatment. This was measured using an electronic pressure pain detector (Somedic, Hörby, Sweden).

Measurement of clinical arthritic score in the rheumatoid arthritis mouse model. On each day of the 5-day treatment, the mice were evaluated for arthritis using a macroscopic scoring system as follows: 11-15, severe arthritis of the entire paw and digits; 6-10, more than two joints involved; $1-5$, two joints involved and 0 , no signs of arthritis.

Measurement of tumor necrosis factor (TNF)- $\alpha$, interleukin (IL)-1 $\beta$ and IL-6. Following the 5-day treatment, blood was collected to evaluate the TNF- $\alpha$, IL- $1 \beta$ and IL- 6 levels with the corresponding enzyme-linked immunosorbent assay kits according to the manufacturer's instructions (R\&D Systems, Minneapolis, MN, USA).

Measurement of nitric oxide (NO) and prostaglandin E2 (PGE2). Following treatment with taraxasterol, mice were sacrificed using cervical vertebra luxation under anesthesia. For the measurement of NO, tissue samples were mixed with Griess reagent and $100 \mu 1$ bronchoalveolar lavage fluid, and then were incubated for $10 \mathrm{~min}$ at room temperature. The optical density at $540 \mathrm{~nm}$ was then measured using a Synergy2 microplate reader (BioTek, Winooski, VT, USA), and the NO level determined by comparison with a standard curve. The PGE2 level was measured using a commercial enzyme immunoassay, with a prostaglandin E metabolite kit (Cayman Chemical, Ann Arbor, MI, USA).

Western blot analysis of cyclooxygenase-2 (COX-2) and nuclear factor $(N F)-\kappa B$. Following the 5-day treatment, paw tissue samples were harvested and frozen in liquid nitrogen immediately prior to homogenization. Protein concentrations were determined using a bicinchoninic acid assay kit (BestBio, Shanghai, China). Equal amounts of protein $(50 \mathrm{mg})$ were separated by $10 \%$ sodium dodecyl sulfate-polyacrylamide gel electrophoresis and subsequently transferred onto a polyvinylidene difluoride (PVDF) membrane (Bio-Rad Laboratories, Inc., Munich, Germany). The PVDF membrane was

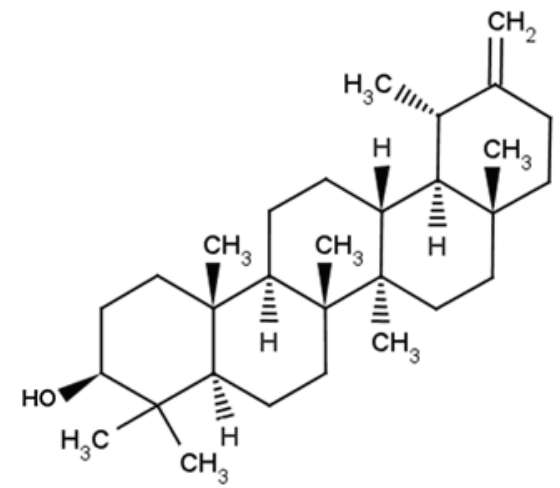

Figure 1. Chemical structure of taraxasterol.

blocked with $5 \%$ skimmed milk in Tris-buffered saline and Tween 20 (TBST) on a shaker for $2 \mathrm{~h}$ at room temperature. The membrane was then incubated with anti-COX-2 (cat. no. sc-514489, 1:1,000; Santa Cruz Biotechnology, Inc., Dallas, TX, USA), anti-NF-кB (cat. no. sc-8008; 1:1,000; American Diagnostica Inc., Stamford, CT, USA) and anti- $\beta$-actin (cat. no. sc-130300; 1:1,000; Santa Cruz Biotechnology, Inc.) primary antibodies. After washing the membrane three times with TBST, the membrane was incubated with secondary antibody (cat. no. sc-2005; 1:5,000; Santa Cruz Biotechnology, Inc.) for $2 \mathrm{~h}$ on the shaker at room temperature. The membrane was then incubated with chemiluminescence reagent (ECL Plus Western Blotting Detection system; GE Healthcare Life Sciences, Chalfont, UK). The relative quantity of the protein was measured using AlphaEase FC (FluorChem FC2) software (Cell Biosciences Inc., Santa Clara, CA, USA).

Statistical analysis. Data are expressed as the mean \pm standard error of the mean. Differences between the mean values of normally distributed data were analyzed using one-way analysis of variance (Dunnett's t-test) and two-tailed Student's t-test. $\mathrm{P}<0.05$ was used to indicate a statistically significant difference.

\section{Results}

Protective effect of taraxasterol against pain in the rheumatoid arthritis mouse model. The protective effect of taraxasterol against pain thresholds in the rheumatoid arthritis mouse model was assessed using an electronic pressure pain detector. As shown in Fig. 2, the pain thresholds in the control group were very similar to those of the taraxasterol only group $(\mathrm{P}>0.05)$ during the entire experiment. By contrast, pain thresholds of the animals in the rheumatoid arthritis group were lower compared with those of the control and taraxasterol only groups after modeling and following treatment for 5 days (Fig. 2). Furthermore, pain thresholds were noticeably increased by treatment with taraxasterol for 5 days in comparison with the rheumatoid arthritis group (Fig. 2).

Protective effect of taraxasterol against clinical arthritis in the rheumatoid arthritis mouse model. The protective effect of taraxasterol in the rheumatoid arthritis mouse model 


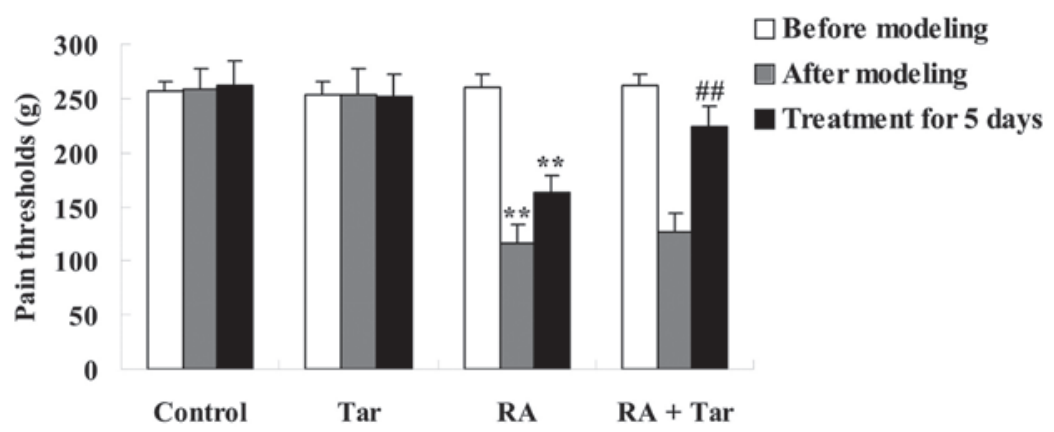

Figure 2. Protective effect of taraxasterol against pain thresholds of a mouse rheumatoid arthritis model. Tar, taraxasterol; RA, rheumatoid arthritis. ${ }^{* *} \mathrm{P}<0.01$ vs. the control group; ${ }^{\# \#} \mathrm{P}<0.01$ vs. the rheumatoid arthritis group.

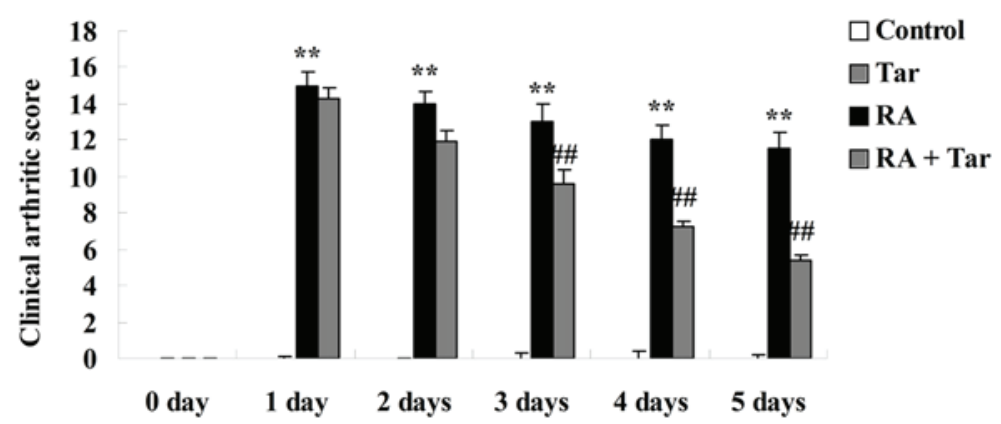

Figure 3. Protective effect of taraxasterol as evaluated using clinical arthritic score in a mouse rheumatoid arthritis model during a 5-day treatment period. Control, control group; Tar, taraxasterol; RA, rheumatoid arthritis. ${ }^{* *} \mathrm{P}<0.01$ vs. the control group; ${ }^{\# \#} \mathrm{P}<0.01$ vs. the rheumatoid arthritis group.

was evaluated using the clinical arthritic score. As shown in Fig. 3, there was no significant difference in the clinical arthritic score between the control and the taraxasterol only groups $(\mathrm{P}>0.05)$. By contrast, rheumatoid arthritis markedly increased the clinical arthritic scores compared with those in the control and taraxasterol only groups (Fig. 3). However, the clinical arthritic score of mice in the rheumatoid arthritis + taraxasterol group was markedly suppressed in comparison with that of the rheumatoid arthritis group (Fig. 3).

Protective effect of taraxasterol against TNF- $\alpha, I L-1 \beta$ and $I L-6$ in the rheumatoid arthritis mouse model. To investigate the protective effect of taraxasterol against inflammation in mice with rheumatoid arthritis, TNF- $\alpha$, IL-1 $\beta$ and IL-6 levels were measured. As shown in Fig. 4, there was no significant difference in the levels of TNF- $\alpha$, IL-1 $\beta$ and IL- 6 between the control and taraxasterol only group $(\mathrm{P}>0.05)$. However, the levels of these inflammatory factors were effectively increased in mice with rheumatoid arthritis compared with the control and taraxasterol only groups (Fig. 4). Furthermore, treatment with taraxasterol decreased the levels of TNF- $\alpha$, IL- $1 \beta$ and IL- 6 in mice with rheumatoid arthritis, when compared with the rheumatoid arthritis group (Fig. 4).

Protective effect of taraxasterol against NO generation in the rheumatoid arthritis mouse model. NO generation was measured to investigate the protective effect of taraxasterol against NO damage in rheumatoid arthritis model mice. As shown in Fig. 5, no significant inter-group difference was identified between the control and taraxasterol only group for $\mathrm{NO}$ generation $(\mathrm{P}>0.05)$. However, there was a significant increase in NO generation in the mice with rheumatoid arthritis compared with the control and taraxasterol only groups (Fig. 5). However, treatment with taraxasterol attenuated the increase in $\mathrm{NO}$ generation in mice with rheumatoid arthritis compared with that in the rheumatoid arthritis group (Fig. 5).

Protective effect of taraxasterol against COX-2 in the rheumatoid arthritis mouse model. In order to define the protective effect of taraxasterol against COX-2 in a rheumatoid arthritis mouse model, COX-2 protein expression levels were examined using western blot analysis. The expression level of COX-2 demonstrated no significant difference between the control and taraxasterol only groups ( $>>0.05$; Fig. 6). However, rheumatoid arthritis noticeably induced the expression of COX-2 protein compared with COX-2 expression levels in the control and taraxasterol only groups (Fig. 6). However, taraxasterol significantly attenuated the change in COX-2 level induced by rheumatoid arthritis (Fig. 6).

Protective effect of taraxasterol against PGE2 in the rheumatoid arthritis mouse model. In order to explore the inhibitory effect of taraxasterol against PGE2 in the rheumatoid arthritis mouse model, the PGE2 level was estimated in the present study. No significant difference was observed in the PGE2 level between the control and taraxasterol only groups ( $\mathrm{P}>0.05$; Fig. 7). The PGE2 level of the rheumatoid arthritis group was higher compared with those of the control or taraxasterol only groups 
A

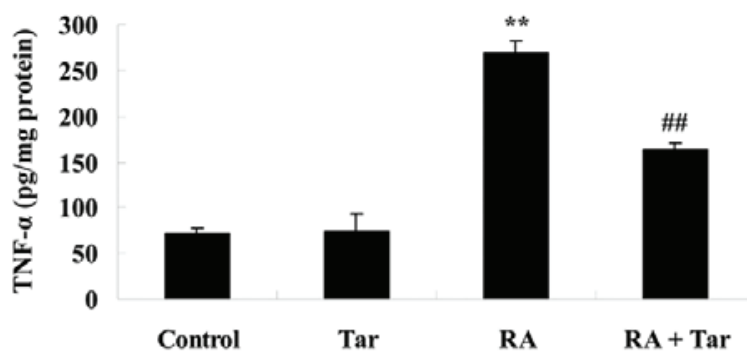

B

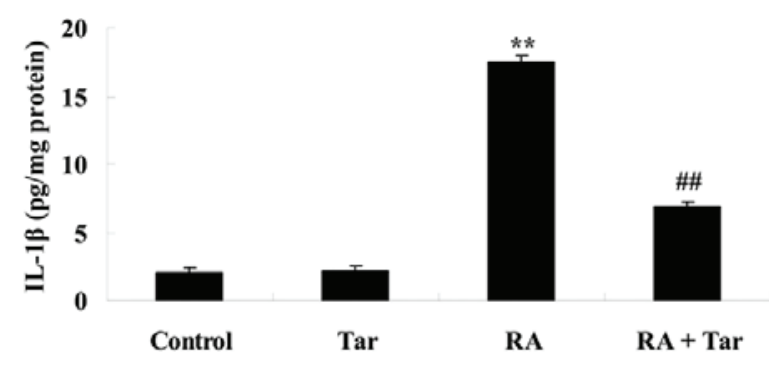

C

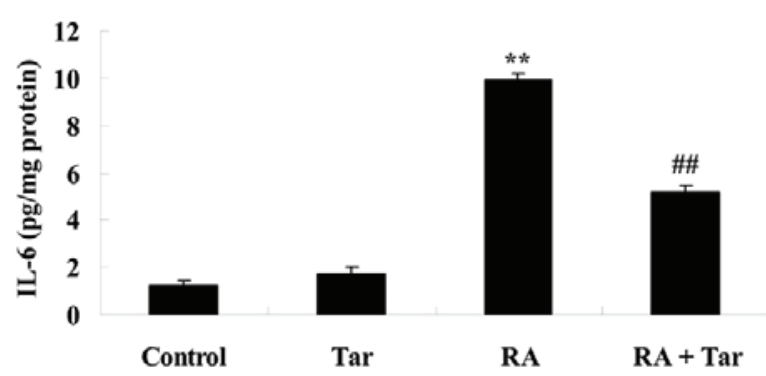

Figure 4. Protective effect of taraxasterol against TNF- $\alpha$, IL-1 $\beta$ and IL-6 in a rheumatoid arthritis mouse model. Protective effect of taraxasterol against (A) TNF- $\alpha$, (B) IL-1 $\beta$ and (C) IL-6 in the model. ${ }^{* *} \mathrm{P}<0.01$ vs. the control group; ${ }^{\# \#} \mathrm{P}<0.01$ vs. the rheumatoid arthritis group. TNF, tumor necrosis factor; Tar, taraxasterol; RA, rheumatoid arthritis; IL, interleukin.

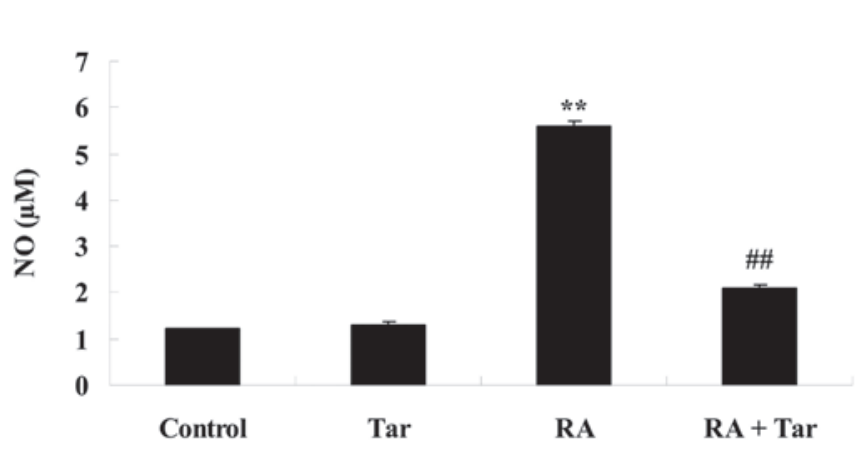

Figure 5. Protective effect of taraxasterol against NO generation in a rheumatoid arthritis mouse model. ${ }^{* *} \mathrm{P}<0.01$ vs. the control group; ${ }^{\# \#} \mathrm{P}<0.01$ vs. the rheumatoid arthritis group. NO, nitric oxide; Tar, taraxasterol; RA, rheumatoid arthritis.

(Fig. 7). However, administration of taraxasterol significantly reduced the PGE2 level in the rheumatoid arthritis mouse model compared with that in the rheumatoid arthritis group (Fig. 7).

Protective effect of taraxasterol against $N F-\kappa B$ in the rheumatoid arthritis mouse model. In order to explore the protective effect of taraxasterol against $\mathrm{NF}-\kappa \mathrm{B}$ in the rheumatoid arthritis mouse model, the protein expression level of $N F-\kappa B$ was measured using western blot analysis. The results of the western blot assay revealed that the protein expression level of NF- $\kappa \mathrm{B}$ in the control group was very similar to that in the taraxasterol only group ( $\mathrm{P}>0.05$; Fig. 8). As shown in Fig. 8, the protein expression of $\mathrm{NF}-\kappa \mathrm{B}$ was significantly increased in the rheumatoid arthritis group compared with the control and taraxasterol only groups (Fig. 8). Furthermore, treatment with taraxasterol significantly decreased the protein expression
A

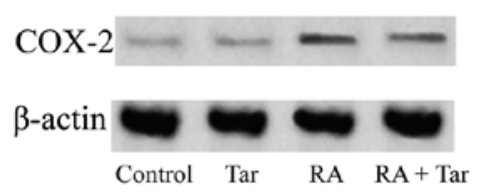

B

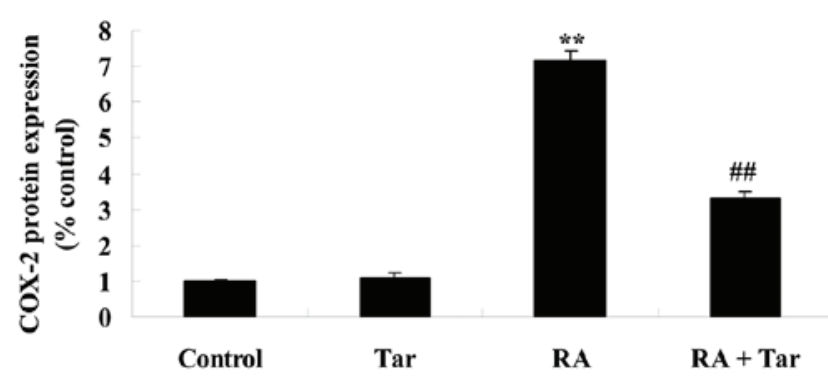

Figure 6. Protective effect of taraxasterol against COX-2 in a rheumatoid arthritis mouse model. (A) Representative western blot showing the protective effect of taraxasterol against COX-2 protein expression and (B) statistical analysis of COX-2 protein expression level in the rheumatoid arthritis mouse model. ${ }^{* *} \mathrm{P}<0.01$ vs. the control group; ${ }^{\# \#} \mathrm{P}<0.01$ vs. the rheumatoid arthritis group. COX-2, cyclooxygenase-2; Tar, taraxasterol; RA, rheumatoid arthritis.

level of NF- $\mathrm{NB}$ in rheumatoid arthritis model mice compared with that in the rheumatoid arthritis group (Fig. 8).

\section{Discussion}

Rheumatoid arthritis is an inflammatory autoimmune disease that involves joints throughout the body. Approximately $1 \%$ of the world's population suffers from this disease, and the morbidity for females is 2-fold higher than that of males (8). Rheumatoid arthritis is distinguished by chronic synovitis of 


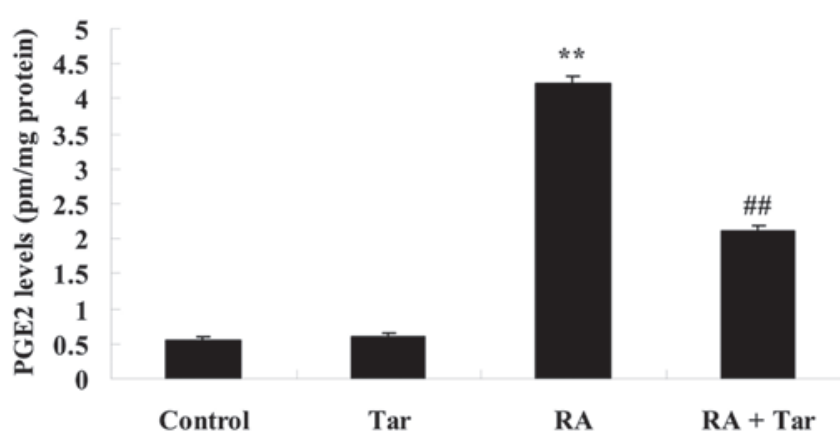

Figure 7. Protective effect of taraxasterol against PGE2 in a rheumatoid arthritis mouse model. ${ }^{* *} \mathrm{P}<0.01$ vs. the control group; ${ }^{\#} \mathrm{P}<0.01$ vs. the rheumatoid arthritis group. PGE2, prostaglandin E2; Tar, taraxasterol; RA, rheumatoid arthritis.

A

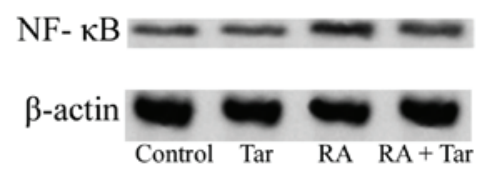

B

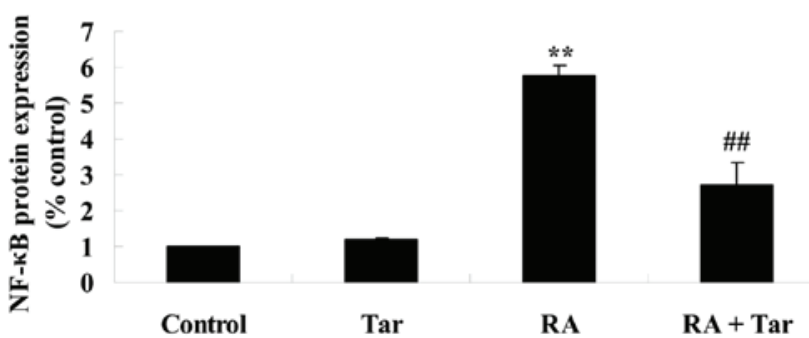

Figure 8. Protective effect of taraxasterol against NF-кB levels in a rheumatoid arthritis mouse model. (A) Representative western blot showing the protective effect of taraxasterol against NF- $\mathrm{KB}$ protein expression and (B) statistical analysis of NF- $\kappa \mathrm{B}$ protein expression level in the rheumatoid arthritis mouse model. ${ }^{* *} \mathrm{P}<0.01$ vs. the control group; ${ }^{\# \#} \mathrm{P}<0.01$ vs. the rheumatoid arthritis group. NF- $\mathrm{kB}$, nuclear factor- $\mathrm{\kappa B}$; Tar, taraxasterol; RA, rheumatoid arthritis

joints and is known to have the following pathological characteristics: Auto-antibody production, synovial cell hyperplasia, inflammatory cell infiltration (such as neutrophile granulocytes and macrophages), pannus production and destruction of bones and cartilage, which finally lead to the destruction and lack of function of a whole joint (9). According to official statistics, rheumatoid arthritis is an important cause of productivity loss and extremity disability in China, with a morbidity rate of $0.32-0.36 \%$ (10). The pathogenesis of rheumatoid arthritis has not been fully elucidated by previous researchers; however, it may be associated with factors including heredity, immunoregulation and the environment (11-13). The present study demonstrated that treatment with taraxasterol significantly increased pain thresholds and inhibited the clinical arthritic scores of mice with rheumatoid arthritis. In a previous study, Zhang et al reported that treatment with taraxasterol protected against LPS-induced endotoxic shock in mice (14). In addition, it appears that taraxasterol may be a useful agent for treating rheumatoid arthritis.
Inflammatory cell infiltration in the joint synovium is one of the key characteristics of rheumatoid arthritis. The infiltrated cells include macrophages, T cells, B cells, dendritic cells and neutrophile granulocytes (15). At the same time, hyperplastic synovial cells invade the cartilago articularis. The aforementioned cells secrete proinflammatory factors and matrix metalloproteinases (MMPs), which can induce aggravation of inflammation and result in the destruction of synovia, cartilage and bones (16). Macrophages around the joint synovium are transformed from mononuclear cells in blood, the process of which is guided by chemotactic factors. Once macrophages become activated, they secrete a mass of inflammatory mediators (including IL-1 $\alpha$, IL-1 $\beta$, IL-6, IL-10, IL-15, IL-17, TNF- $\alpha$ and granulocyte-macrophage colony-stimulating factor), proinflammatory factors, growth factors, chemotactic factors and MMPs (17). All these factors can cause increased inflammation and play a main role in injury of the synovium and joints (18). In the present study, it was identified that treatment with taraxasterol significantly suppressed TNF- $\alpha$, IL-1 $\beta$, and IL-6 levels and the protein expression of $\mathrm{NF}-\kappa \mathrm{B}$ in mice with rheumatoid arthritis. Zhang et al reported that the effects of taraxasterol protect LPS-treated RAW 264.7 macrophages through suppression of the inflammatory response (19). Piao et al demonstrated that taraxasterol protects human osteoarthritic chondrocytes by inhibition of IL-1 $\beta$-induced inflammatory response (6). Therefore, the anti-inflammatory effect of taraxasterol may have potential in preventing rheumatoid arthritis.

Activated inducible nitric oxide synthase (iNOS), an important inflammatory mediator, can produce a large quantity of NO that inhibits DNA synthesis, induces cell apoptosis and causes cytotoxic effects by restraining the Kreb's cycle (20). PGE2 is another inflammatory mediator, trace amounts of which can lead to intense inflammation; therefore, PGE2 is important in physiological and pathological processes (21). PGE2 is generated by continuous enzymatic reactions as follows: Arachidonic acid is released from the membrane phospholipid by catalysis of phospholipase A2, prostaglandin $\mathrm{H} 2$ (PGH2) is generated from arachidonic acid by catalysis with COX and finally PGE2 is created from $\mathrm{PGH} 2$ by catalysis with prostaglandin E synthase (PGES) (22). The expression of membrane-bound PGES-1 and COX-2 and the production of PGE2 are increased by inflammatory factors. COX-2 is an important enzyme in the development of inflammation; its expression levels are low under normal conditions, but are increased strongly in the presence of LPS (23). According to a previous study, the severity of rheumatoid arthritis can be reduced in a dose-dependent manner by suppressing the expression of NOS, COX-2 and PGE2 (24). In the present study, NO, PGE2 and COX-2 protein expression levels were significantly reduced by treatment with taraxasterol in a mouse model of rheumatoid arthritis. Furthermore, Xiong et al indicated that taraxasterol treatment weakens LPS-induced effects on RAW 264.7 macrophages and reduces iNOS and COX-2 expression (25). In addition, Piao et al revealed that taraxasterol suppresses PGE2 and NO production in human osteoarthritic chondrocytes (6). The effect of taraxasterol against iNOS, COX-2 and PGE2 pathways may support its consideration as a potential agent for the treatment of rheumatoid arthritis.

In conclusion, the present study revealed a protective effect of taraxasterol against rheumatoid arthritis, which is mediated 
by the modulation of inflammatory responses and the iNOS, COX-2 and PGE2 pathways in mice. These results suggest that taraxasterol may be a potential protective agent against rheumatoid arthritis.

\section{References}

1. Vogel WV, van Riel PL and Oyen WJ: FDG-PET/CT can visualise the extent of inflammation in rheumatoid arthritis of the tarsus. Eur J Nucl Med Mol Imaging 34: 439, 2007.

2. Bijlsma JW, Welsing PM, Woodworth TG, Middelink LM, Pethö-Schramm A, Bernasconi C, Borm ME, Wortel CH, ter Borg EJ, Jahangier ZN, et al: Early rheumatoid arthritis treated with tocilizumab, methotrexate, or their combination (U-Act-Early): A multicentre, randomised, double-blind, double-dummy, strategy trial. Lancet 388: 343-355, 2016.

3. Hao GF, Li YS, Liu JL and Wo MY: Association of HLA-DQA1 (rs9272219) with susceptibility to rheumatoid arthritis in a Han Chinese population. Int J Clin Exp Pathol 7: 8155-8158, 2014.

4. Majumdar KN, Banerjee A, Ratha J, Mandal M, Sarkar RN and Saha KD: Leishmanial lipid suppresses tumor necrosis factor alpha, interleukin-1beta, and nitric oxide production by adherent synovial fluid mononuclear cells in rheumatoid arthritis patients and induces apoptosis through the mitochondrial-mediated pathway. Arthritis Rheum 58: 696-706, 2008

5. Herz W and Mirrington RN: Identification of pyrethrol with taraxasterol. J Pharm Sci 55: 104, 1966.

6. Piao T, Ma Z, Li X and Liu J: Taraxasterol inhibits IL-1 $\beta$-induced inflammatory response in human osteoarthritic chondrocytes. Eur J Pharmacol 756: 38-42, 2015.

7. Luo L, Hu L, He L, Tang ZL, Song XG, Dirckinck-Holmfeld L and Cai RL: Effect of moxibustion on ultrastructure of synovial cells in rheumatoid arthritis rats. Zhen Ci Yan Jiu 36 105-109, 2011 (In Chinese).

8. Ahmed S, Anuntiyo J, Malemud CJ and Haqqi TM: Biological basis for the use of botanicals in osteoarthritis and rheumatoid arthritis: A review. Evid Based Complement Alternat Med 2: 301-308, 2005.

9. Li J, Zhou T and Zhao F: Inhibitory effect of sodium houttuyfonate on synovial proliferation in vitro in cells from a patient with rheumatoid arthritis. Exp Ther Med 7: 1639-1642, 2014.

10. Jiang P, Li H and Li X: Diabetes mellitus risk factors in rheumatoid arthritis: A systematic review and meta-analysis. Clin Exp Rheumatol 33: 115-121, 2015.

11. Sparks JA and Costenbader KH: Genetics, environment, and gene-environment interactions in the development of systemic rheumatic diseases. Rheum Dis Clin North Am 40: 637-657, 2014.

12. Wen H and Baker JF: Vitamin D, immunoregulation, and rheumatoid arthritis. J Clin Rheumatol 17: 102-107, 2011.
13. Souliotis K, Papageorgiou M, Politi A, Ioakeimidis D and Sidiropoulos P: Barriers to accessing biologic treatment for rheumatoid arthritis in Greece: The unseen impact of the fiscal crisis - The Health Outcomes Patient Environment (HOPE) study. Rheumatol Int 34: 25-33, 2014.

14. Zhang X, Xiong H, Li H and Cheng Y: Protective effect of taraxasterol against LPS-induced endotoxic shock by modulating inflammatory responses in mice. Immunopharmacol Immunotoxicol 36: 11-16, 2014.

15. Kikuchi J, Hashizume M, Kaneko Y, Yoshimoto K, Nishina N and Takeuchi T: Peripheral blood CD4 (+) CD25 (+) CD127 low regulatory $\mathrm{T}$ cells are significantly increased by tocilizumab treatment in patients with rheumatoid arthritis: Increase in regulatory $\mathrm{T}$ cells correlates with clinical response. Arthritis Res Ther 17: 10, 2015.

16. Tetlow LC and Woolley DE: The effects of 1 alpha, 25-dihydroxyvitamin $\mathrm{D}(3)$ on matrix metalloproteinase and prostaglandin $\mathrm{E}(2)$ production by cells of the rheumatoid lesion. Arthritis Res 1: 63-70, 1999.

17. Hein GE, Köhler M, Oelzner P, Stein G and Franke S: The advanced glycation end product pentosidine correlates to IL-6 and other relevant inflammatory markers in rheumatoid arthritis. Rheumatol Int 26: 137-141, 2005.

18. Ma X and Xu S: TNF inhibitor therapy for rheumatoid arthritis. Biomed Rep 1: 177-184, 2013.

19. Zhang X, Xiong H and Liu L: Effects of taraxasterol on inflammatory responses in lipopolysaccharide-induced RAW 264.7 macrophages. J Ethnopharmacol 141: 206-211, 2012.

20. Chenevier-Gobeaux C, Simonneau C, Lemarechal H, Bonnefont-Rousselot D, Poiraudeau S, Rannou F, Anract P and Borderie D: Hypoxia induces nitric oxide synthase in rheumatoid synoviocytes: Consequences on NADPH oxidase regulation. Free Radic Res 46: 628-636, 2012.

21. Hishinuma T, Nakamura H, Sawai T, Uzuki M, Itabash Y and Mizugaki M: Microdetermination of prostaglandin E2 in joint fluid in rheumatoid arthritis patients using gas chromatography/selected ion monitoring. Prostaglandins Other Lipid Mediat 58: 179-186, 1999.

22. Lee WS, Lim JH, Sung MS, Lee EG, Oh YJ and Yoo WH: Ethyl acetate fraction from Angelica sinensis inhibits IL-1 $\beta$-induced rheumatoid synovial fibroblast proliferation and COX-2, PGE2, and MMPs production. Biol Res 47: 41, 2014.

23. Sung MS, Lee EG, Jeon HS, Chae HJ, Park SJ, Lee YC and Yoo WH: Quercetin inhibits IL-1 $\beta$-induced proliferation and production of MMPs, COX-2, and PGE2 by rheumatoid synovial fibroblast. Inflammation 35: 1585-1594, 2012

24. Lee EG, Lee SL, Chae HJ, Park SJ, Lee YC and Yoo WH: Ethyl acetate fraction from Cudrania tricuspidata inhibits IL-1 $\beta$-induced rheumatoid synovial fibroblast proliferation and MMPs, COX-2, and PGE2 production. Biol Res 43: 225-231, 2010.

25. Xiong $\mathrm{H}$, Cheng Y, Zhang X and Zhang X: Effects of taraxasterol on iNOS and COX-2 expression in LPS-induced RAW 264.7 macrophages. J Ethnopharmacol 155: 753-757, 2014. 